\title{
Construcción, validación y confiabilidad de escala de medición de deserción estudiantil universitaria
}

\author{
Construction, validation and reliability of a scale to measure the retention \\ of college students
}

\section{ISSN 2071-8748 \\ E-ISSN 2218-3345 \\ (c) (1) $\$(2)$ \\ BY NC SA}

\author{
José Modesto Ventura-Romero' \\ ORCID: 0000-0003-3641-0442 \\ Marlon Elías Lobos-Rivera ${ }^{2}$ \\ José Ricardo Gutiérrez-Quintanilla ${ }^{3}$
}

Recibido: 21 de noviembre 2018 Aprobado: 4 de abril 2019

URI: http://hdl.handle.net/11298/964

\section{Resumen}

El objetivo del presente estudio es la construcción, validación y confiabilidad de una escala para medir la deserción estudiantil universitaria. El estudio es de tipo instrumental. El muestreo es no probabilístico; la muestra estaba conformada por 361 estudiantes universitarios que hubieran desertado una o más veces. La media de edad general es de 28.35 años. La muestra de mujeres fue de 181 (50,1\%), y la de hombres fue de $180(49,9 \%)$. La técnica utilizada fue la encuesta. Los instrumentos aplicados fueron la escala de deserción estudiantil universitaria y el cuestionario de adaptación a la vida universitaria. En los resultados, se tiene que la prueba posee adecuados índices de validez de contenido, de constructo, de criterio divergente y confiabilidad para medir deserción estudiantil universitaria.

\section{Abstract}

The aim of this study is the construction, validation and reliability of a scale to measure the number of students who drop out from their college studies. This is an instrumental study with a non-probabilistic sampling; the sample consisted of 361 college students who had dropped out one or more times. The overall average age is 28.35 years. The sample included 181 women (50.1\%) and 180 men (49.9\%). The technique used was the survey. The instruments used were the College Retention Scale and the Student Adaptation to College Questionnaire. Results demonstrated that the test showed adequate indices of validity in terms of content and construct; the criteria was divergent and reliable for the measurement of the retention of university students.

\footnotetext{
1 José Modesto Ventura-Romero. Es Doctorando en Educación. Vicerrector Académico de la Universidad Tecnológica de El Salvador. jose.ventura@utec.edu.sv 2 Marlon Elías Lobos-Rivera. Es Licenciado en Psicología. Profesor asociado e investigador. marlon.lobos@mail.utec.edu.sv

3 José Ricardo Gutiérrez-Quintanilla. Es Doctor en Psicología. Profesor titular e investigador. Universidad Tecnológica de El Salvador. jose.gutierrez@utec.edu.sv
} 


\section{Palabras clave}

Deserción universitaria-El Salvador-mediciones- investigaciones, asistencia a la universidad-mediciones, deserción universitaria-El Salvador-mediciones-estudio de casos, medición por escalas (ciencias sociales).

\section{Keywords}

College retention-El Salvador-measurements-research; college attendance-measurements; college dropouts-El Salvador- measurements-case studies; measuring scales (Social Sciences).

\section{Introducción}

Antecedentes de la deserción. En Fiegehen González (2006) [Chile], se expresa que la desigualdad de acceso a las instituciones de educación superior (IES) se mantiene en la mayoría de los países latinoamericanos como una condición de exclusión, para una proporción mayoritaria y significativa de la población de cada país. El informe evidenció que menos del 20,0 \% de los adultos mayores de 25 años ha accedido a la universidad, y que menos de un $10,0 \%$ ha completado sus estudios universitarios. También, se analizó la deserción por carreras específicas, encontrando que en la de Derecho, la deserción promedio en América Latina es de un 40,0 \%; en la de Medicina es de un 32,1\%, y en la de Ingeniería civil es de un $37,7 \%$. Las estadísticas chilenas sobre la deserción estudiantil universitaria presentan la tasa de deserción global de pregrado evaluada en el 2004, cercana al 53,7 \%, siendo mayor en las universidades privadas nuevas que en las públicas. Por otra parte, las áreas de conocimiento más críticas son Humanidades y Derecho, con cifras aproximadamente del $80 \%$; y las más eficientes son las áreas de Educación, con un 37.0\%; y Salud, con un $27,0 \%$. Según el género, las mujeres poseen una tasa de deserción promedio más baja en comparación con los varones, siendo de 43,0 y $50,0 \%$, respectivamente (González, 2005).

En Colombia, Rojas-Betancur (2008) encontró que la más alta deserción se da en los hombres, conformando el 62,0\%, y las mujeres, el $38,0 \%$. Este autor expresa que las mujeres tienen un mayor nivel de estabilidad en la universidad en comparación con los hombres. En relación con las actividades económicas, un $24,0 \%$ de la muestra respondió que solo se dedica a trabajar; $10,0 \%$ dice que se dedica al hogar, y un $14,0 \%$ dice que combina ambas actividades. El $48,0 \%$ del total de los desertores responde que se dedican de forma exclusiva al estudio. En la Universidad Tecnológica de El Salvador (Utec), durante el ciclo 02-2018, han realizado trámites de retiro de ciclo 890 estudiantes, de los cuáles el
$37,1 \%$ son de las carreras de Ciencias Empresariales, el $18,3 \%$ de Ciencias Sociales, el $10 \%$ de Ciencias Jurídicas y el $34,6 \%$ de Informática y Ciencias Aplicadas. Las principales causas de retiro son los siguientes: factores laborales (49\%), factores económicos $(27,8 \%)$, problemas familiares y personales $(6,2 \%)$, lo obligan a salir del país $(8,1 \%)$, y problemas académicos y de adaptación (8,9\%).

Teniendo como meta la construcción de un instrumento para medir la deserción universitaria en El Salvador, se realizó una revisión bastante exhaustiva de la literatura relativa a la deserción estudiantil de la universidad; y la búsqueda de instrumentos que midiesen este problema educativo. Paradójicamente a las expectativas del equipo, no se encontraron instrumentos válidos y fiables para medir la deserción estudiantil en las IES. Debido a que el primer autor de este artículo está realizando su tesis doctoral en Educación, surge la necesidad de construir una prueba que midiese los factores relacionados con la deserción estudiantil en la universidad. Para ello, se consideró pertinente la operacionalización en indicadores la propuesta de cuatro factores que sugiere Álvarez (1997) para medir la deserción estudiantil universitaria, que son: personales, académicos, socioeconómicos e institucionales. A este modelo se le agregó un quinto factor, que es el sociocultural, excluyéndose el institucional por encontrarse inmerso en el factor académico, aplicándose el modelo propuesto por Álvarez modificado. Tras la obtención de los indicadores, se construyó un universo de 37 reactivos e ítems que representa los cuatro factores señalados. En un segundo momento, se solicitó a 10 profesores universitarios, con experiencia y formación docente, que revisaran los reactivos en relación con el factor estudiado y que brindaran su opinión en cada ítem (en desacuerdo, moderado acuerdo y de acuerdo). Estas opiniones fueron procesadas en el paquete estadístico SPSS versión 22.0, y se aplicó el método de correspondencia inter-evaluador (índice de concordancia de Kendal), obteniéndose un coeficiente estadístico de .80, indicando que la prueba propuesta posee buena validez 
de contenido. El presente estudio tiene como objetivo construir, validar y fiabilizar una escala que mida los factores relacionados con la deserción estudiantil universitaria, con la finalidad de determinar y clasificar los factores asociados con la deserción: socioeconómicos, sociodemográficos, de personalidad, culturales y académicos.

Hablar de la deserción estudiantil en educación superior significa enfocarse en aquellas variables asociadas con posibles causales del fenómeno, unas con menor grado de incidencia que otras. Páramo y Correa (1999) plantean que la deserción estudiantil se entiende como el abandono definitivo de las aulas de clase por diferentes razones; es la no continuidad de la formación académica que la sociedad quiere que termine. La Universidad de Uruguay (2003) define el término deserción como el proceso de abandono voluntario o forzoso de la carrera en la que se matricula un estudiante, por la influencia positiva o negativa de circunstancias internas o externas al estudiante. Por otra parte, Abarca-Rodríguez y Sánchez-Vindas (2005) expresan que la deserción universitaria es una manifestación de conducta que podría ser el resultado de la interacción de una serie de características o variables que reviste una implicación fundamental, que refleja la decisión de un individuo, de un estudiante universitario, de interrumpir sus actividades académicas. En esta misma línea, Himmel (2005) describe la deserción como el abandono prematuro de un programa de estudios antes de alcanzar el título o grado; y considera un tiempo suficientemente largo para descartar la posibilidad de que el estudiante se reincorpore al sistema educativo. Otra definición de deserción estudiantil es la propuesta por Rodríguez y Hernández (2008), quienes mencionan que la deserción estudiantil puede entenderse como la ruptura del vínculo de la matrícula académica del estudiante con la universidad, la cual tiene efectos financieros graves, académicos y sociales recíprocos.

Pérez, Bravo e Isabeles (2008) consideran desertor al alumno de nivel medio superior de una carrera o de posgrado que no se inscribe en las fechas correspondientes al plan de estudios de su cohorte, ni se reinscribe en períodos ya cursados. Según González-Fiegehen y Espinoza-Díaz (2008), la deserción en la educación superior se puede definir como la cantidad de estudiantes que abandonan el sistema de educación superior entre uno y otro período académico, especialmente por semestre o año. Para Mateus-Rodríguez, Herrera-Hernández, Perilla-Suárez, Parra-Quecan, y veraMaldonado (2011), la deserción se refiere al abandono prematuro de un programa de estudios antes de alcanzar $u$ obtener el título o grado académico, y consideran un tiempo suficientemente largo como para descartar la posibilidad de que el estudiante se reincorpore. Mori-Sánchez (2012) define la deserción estudiantil como el abandono definitivo o temporal, voluntario o forzado, que se puede distinguir por diferentes modalidades, tales como el abandono de la carrera, abandono de la institución y el abandono del sistema de educación superior.

Tipos de deserción. Dentro de la deserción estudiantil universitaria existen diferentes tipos, entre ellos tenemos: la deserción total, la cual se entiende como el abandono definitivo de la formación académica individual; la deserción discriminada por causas, la cual se da según la causa de la deserción, por ejemplo, problemas de salud, financieros, conflictos entre compañeros o docentes, entre otros; la deserción por facultad, que se refiere al cambio de facultad, escuela o departamento, por ejemplo, cuando el estudiante se cambia de carrera y asiste a una facultad diferente; deserción por programa, la cual consiste en el cambio de un programa, pero el estudiante sigue asistiendo a la misma facultad; la deserción a primer semestre (ciclo) de carrera, la cual surge por una inadecuada adaptación a la vida universitaria; y la deserción acumulada, la cual se refiere a la sumatoria de deserciones de una institución (Páramo \& Correa, 1999, p. 68).

Para Bolaños (1985, p. 11), la deserción se divide en dos tipos: la deserción intra-semestral, la cual ocurre en el caso de retiros justificados o injustificados durante el semestre; y la deserción inter-semestral, la cual se da con el cese de matrícula para un determinado semestre. Para Durán y Díaz (1990), existen tres modalidades de deserción: la deserción voluntaria, la cual se da cuando el estudiante realiza el procedimiento establecido por la administración escolar denominado baja definitiva o baja voluntaria; la deserción incurrida, que sucede cuando el estudiante viola las disposiciones reglamentarias de la institución educativa; y la deserción potencial, que pasa cuando el estudiante no ha realizado trámites académico-administrativos durante 7 o más trimestres consecutivos y tiene menos de 35 créditos académicos. La deserción también puede ser clasificada de forma categórica. Cabrera, Bethencourt, Álvarez-Pérez y González-Afonso (2006) mencionan que la deserción estudiantil tiene siete categorías: 1. abandono involuntario por incumplimiento administrativo o violación de los reglamentos institucionales, 2. dejar la carrera para 
iniciar otra en la misma institución, 3. dejar la carrera para iniciar otra en otra institución, 4. dejar la universidad e irse a otra para completar los estudios iniciados, 5. renunciar a la formación universitaria para iniciar itinerarios formativos fuera de la universidad o incorporarse al mundo laboral y 6. interrumpir la formación con la intención de retomarla en el futuro.

Características del estudiante desertor. Son aquellas características que presenta un estudiante universitario que puedan dar origen al pensamiento o acción de desertar de sus estudios y por consiguiente un riesgo de deserción. Páramo y Correa (1999) aportan características que tienen los estudiantes desertores o que están en riesgo de desertar de la institución universitaria: bajo aprovechamiento de oportunidades educativas, problemas de disciplina, hijos de padres que no les interesa la educación, problemas con la justicia, adolecen de motivación e interés para realizar su labor educativa, nivel socioeconómico bajo o sin opción económica, ausentismo a clases, problemas de salud psicosomática, problemas inherentes a la edad, inadecuadas relaciones interpersonales, provienen de ambientes familiares y sociales violentos, baja empatía por el trabajo de sus pares, resistencia a desarrollar actividades formativas, inapetencia por el conocimiento y desmotivación hacia la carrera y a la universidad.

Factores y variables asociadas a la deserción. La deserción estudiantil tiene factores o variables asociadas que pueden impactar en menor o mayor medida e intensidad. Páramo y Correa (1999) señalan algunas variables que están asociadas con la deserción estudiantil, entre ellas están: los ambientes educativos universitarios en los cuales está inmerso el estudiante; el proceso educativo y acompañamiento al estudiante en su formación; la edad, debido a que la mayoría de estudiantes universitarios son muy jóvenes; la adaptación social del estudiante desertor con sus pares; bajos niveles de comprensión unidos a la falta de interés y apatía por programas curriculares; modelos pedagógicos universitarios diferentes a los modelos de bachillerato, que imprimen una alto nivel de exigencia; programas microcurriculares universitarios rígidos con respecto a los de su formación secundaria, de alta intensidad temática, dispuestos en corto tiempo; evaluaciones extenuantes y avasalladoras, debido al nivel de complejidad que presentan las evaluaciones $y$ trabajos en la universidad; cursos no asociados con, ni aplicables a, su ejercicio profesional; factores económicos que impiden la continuidad del desertor en la universidad; cantidad de oferentes académicos; orientación profesional, y masificación de la educación.

Causas de la deserción. González-Fiegehen y Espinoza-Díaz (2008) determinan que las causas de la deserción pueden ser externas, propias del sistema, e institucionales, de orden académico y personales. Entre las principales causas externas están: las condiciones socioeconómicas tanto del estudiante como del grupo familiar (lugar de residencia, nivel de ingresos, nivel educativo de los padres, el ambiente familiar, la necesidad de incursionar en el campo laboral para mantenerse o aportar económicamente a su familia).

Entre las causas propias del sistema e institucionales se encuentran: el incremento de la matrícula; la carencia de mecanismos adecuados de financiamiento del sistema, en especial para el otorgamiento de ayudas estudiantiles, créditos y becas; las políticas de admisión (sean estas abiertas o selectivas); el desconocimiento de la profesión y de la metodología de las carreras; el ambiente educativo e institucional; y la carencia de lazos afectivos con la institución de educación superior.

En las causas de orden académico están: la formación académica previa, los exámenes de ingreso, el nivel de aprendizaje que adquiere el estudiante, la excesiva orientación teórica y la escasa vinculación de los estudios con el mundo laboral, la falta de apoyo y orientación recibida por los profesores, la falta de información al elegir la carrera, la carencia de preparación de los profesores para enfrentar la población estudiantil.

Entre las causas personales se consideran: la actividad económica del estudiante, sus aspiraciones y motivaciones personales, la disonancia con sus expectativas, insuficiente madurez emocional, aptitudes propias de la juventud, el grado de satisfacción con la carrera, las expectativas al egreso de la carrera en relación con el mercado laboral, dificultades personales para la integración y adaptación, la dedicación del estudiante, falta de aptitudes y habilidades o interés por la carrera escogida.

Según Álvarez (1997), las causas de la deserción estudiantil universitaria se asocian con los siguientes factores: 1. Factores personales, que están compuestos por motivos psicológicos que comprenden aspectos motivacionales, emocionales, desadaptación e insatisfacción de expectativas; motivos sociológicos debidos a influencias familiares y de otros 
grupos como los amigos o vecinos; y otros motivos no clasificados como edad, salud, fallecimiento, entre otros. 2. Factores académicos: entre estos están los problemas cognitivos como bajo rendimiento académico, repitencia, ausencia de disciplina y métodos de estudio; deficiencias universitarias como dificultades en los programas académicos que tienen que ver con la enseñanza tradicional, insatisfacción académica generada por la falta de espacios pedagógicos adecuados para el estudio, falta de orientación profesional y falta de aptitud académica. 3. Factores socioeconómicos: bajos ingresos familiares, desempleo, falta de apoyo familiar, incompatibilidad de horario entre trabajo y estudio. 4. Factores institucionales: causados por el cambio de institución, deficiencia administrativa, influencia negativa de los docentes y personas involucradas con la institución, programas académicos obsoletos y rígidos, y baja calidad educativa.

\section{Método}

\section{Participantes}

El presente estudio es de tipo instrumental con un diseño retrospectivo (Montero \& León, 2007). Se seleccionó una muestra no probabilística, intencionada, de 361 estudiantes de la Universidad Tecnológica de El Salvador, que han desertado al menos una vez de la institución. La muestra de mujeres fue de $181(50,1 \%$ y la de hombres fue de 180 $(49,9 \%)$. La media general de edad es de 28.35 ( $D E=7.57$ ). La media de edad de mujeres fue 27.74 años $(D T=6.53)$ y la de hombres fue de 28.97 años $(D T=8.46)$. La técnica utilizada en la recolección de la información fue la encuesta.

\section{Instrumentos}

Escala de deserción estudiantil universitaria (EDEU). Esta escala fue construida por el equipo investigador a partir de la propuesta de cuatro factores de Álvarez (1997) y de diversas perspectivas teóricas relacionadas con la deserción (Ariza-Gasca \& Marín-Arias, 2009; Montero-Rojas, VillalobosPalma \& Valverde-Bermúdez, 2007; Latiesa, 1992); está conformada por 37 reactivos o ítems, los cuales pretenden medir cuatro dimensiones que pretenden medir las posibles causas por las cuales el/la estudiante ha decidido desertar de sus estudios universitarios, las cuales son: factores socioeconómicos (ítems $1,2,3,4,5,6,7,8,9,10$ ); factores de personalidad (ítems 11, 12, 13, 14, 15, 16, 17, 18, 19); factores socioculturales (ítems 20, 21, 22, 23, 24, 25, 26); y factores académicos (ítems 27, 28, 29, 30, 31, 32, 33, $34,35,36,37)$; así también, el instrumento cuenta con una escala de respuesta de tipo Likert compuesta por cinco opciones de respuesta, desde el ítem 1 hasta el 26 tienen como respuesta: $1=$ Nunca, $2=$ Rara vez, $3=$ Algunas veces, $4=$ Casi siempre, y $5=$ Siempre, y del ítem 27 hasta el 37 tienen como respuesta: $1=$ Deficiente, $2=$ Regular, $3=$ Bueno, 4= Muy bueno, y $5=$ Excelente.

Cuestionario de adaptación a la vida universitario (CAVU). Este cuestionario es una versión chilena realizada por Rodríguez-Ayan y Sotelo (2014). Esta versión está conformada por 11 reactivos que evalúan la adaptación del estudiante dentro del contexto educativo universitario; y se le aplicó el análisis factorial exploratorio, el cual explica la suma de tres factores con el $55,4 \%$ de la varianza, las dimensiones son: adaptación social (ítems 1, 2, 3, 4, 5) con un Alfa de Cronbach de .77; adaptación institucional (ítems 6, 7, 8) con un Alfa de Cronbach de .59; y adaptación académica (ítems 9, 10, 11) con un Alfa de Cronbach de .59. También aplicaron un análisis factorial confirmatorio, en el cual se estimaron los parámetros del modelo de tres factores relacionados; el resultado fue consistente con el modelo de covarianzas estructurales $(p=.821)$. Para el presente estudio, se replicaron los análisis de fiabilidad mediante el Alfa de Cronbach; para la dimensión adaptación social, se obtuvo un alfa de Cronbach de .77; para la dimensión adaptación institucional, se obtuvo un alfa de Cronbach de .85; y para la dimensión adaptación académica, se obtuvo un Alfa de Cronbach de .84 .

\section{Resultados}

A continuación, se presentan los análisis de resultados, aplicándose los análisis psicométricos de validez y confiabilidad obtenidos a partir de la muestra evaluada. Con respecto a la validez, se utilizaron tres tipos. El primero: la validez de contenido mediante el método de concordancia de Kendal, o llamado de expertos, brindando un coeficiente de concordancia interevaluador de .80 , indicando que la prueba tiene adecuada validez de contenido para medir la deserción estudiantil. El segundo: el análisis factorial exploratorio (AFE), mediante componentes principales para comprobar empíricamente la estructura factorial de la prueba, como indicador de validez de constructo. El tercero: la validez de criterio de tipo discriminante, o también llamada divergente. También se aplicará el análisis de confiabilidad mediante el análisis de consistencia interna (Alfa de Cronbach), y de esta 
manera comprobar si el instrumento es válido y fiable para utilizarlo en estudios futuros.

\section{Validez de contenido}

Para realizar la validez de contenido, se contactó con 10 expertos, a quienes se les solicito su colaboración y explicó con detalle acerca de qué trataba el instrumento. También se explicó que su aporte consistía en asegurar la redacción adecuada de los ítems y que realmente cada pregunta midiese el factor que pretende medir. Se les entregó impresas las definiciones de cada dimensión, el instrumento completo y una plantilla de evaluación en donde cada experto brindó su respectiva evaluación a cada pregunta, según la dimensión a la que correspondía el ítem. La plantilla contenía el constructo (en este caso es la deserción universitaria), las cuatro dimensiones de la escala y el número de la pregunta; cada pregunta debía ser respondida mediante una de las tres respuestas posibles, donde $1=$ en desacuerdo, $2=$ moderado acuerdo y $3=$ de acuerdo.

Después de tener las respuestas de los 10 expertos, se procesaron en el programa estadístico SPSS versión 22.0; luego se aplicó el método de concordancia inter-evaluador de Kendall $(W)$ para determinar si el instrumento presenta una adecuada validez de contenido. Como resultado, el instrumento presentó un índice de concordancia adecuado $(w=.80)$, indicando que los 10 expertos consultados coincidieron mayoritariamente en la redacción de los ítems y las dimensiones que busca medir, indicando que el instrumento presenta una adecuada validez de contenido.

\section{Validez de constructo}

La escala de factores asociados con la deserción estudiantil universitaria fue diseñada por el equipo investigador el presente año, operativizadas bajo cuatro dimensiones que explican las posibles causas de deserción universitaria. La escala que pretende medir los factores que influyen en la deserción estudiantil universitaria y está construida por 37 reactivos, divididos en 4 dimensiones: 1) factores socioeconómicos, 2) factores de personalidad, 3) factores socioculturales y 4) factores académicos. La primera dimensión está conformada por 10 ítems, la segunda con 9, la tercera con 7 y la cuarta con 11.

Tras la administración del universo de ítems a una muestra de estudiantes desertores, los datos fueron procesados en el programa estadístico SPSS. En la segunda fase de este proceso, se realizó un análisis factorial exploratorio mediante el método de componentes principales. Tanto el análisis Kaiser-Meyer-Olkin $(K M O=.84)$ como la prueba de esfericidad de Bartlett $\left(X^{2}{ }_{361}=5253.80 ; p=.001\right)$ indicaron la adecuación de los datos para este tipo de análisis. Siguiendo el procedimiento de factores con rotación Varimax, resultó en una solución de cuatro factores que explican el $44,41 \%$ de la varianza, presentando la mayoría de los ítems valores de saturación superiores a .40; a excepción de los reactivos $8,9,10,24,25$ y 26, los cuales tuvieron una carga factorial inferior a .40. Razón por la que fueron excluidos de la prueba.

En la tabla 1 se presenta la solución factorial obtenida y el porcentaje de la varianza explicada por cada factor. En este sentido, se describe cada una de las dimensiones o factores de la escala: la dimensión factores socioeconómicos está conformado con 9 ítems $(1,2,3,4,5,6,7,20$, 21); la dimensión factores de personalidad está integrado con 7 ítems $(11,12$, $13,14,15,16,17)$; la dimensión factores socioculturales lo conforman 4 ítems $(18,19,22,23$,); y la dimensión factores académicos lo conforman once ítems $(27,28,29,30,31,32$, $33,34,35,36,37)$. 
Tabla 1. Matriz de componentes principales (factores), su carga factorial rotada y el porcentaje de varianza total explicada por cada dimensión de la escala de deserción estudiantil universitaria

\begin{tabular}{|c|c|c|c|c|}
\hline \multirow[b]{2}{*}{ Reactivos } & \multicolumn{4}{|c|}{ Factores/Componentes } \\
\hline & $\begin{array}{c}\text { Factores } \\
\text { académicos }\end{array}$ & $\begin{array}{c}\text { Factores } \\
\text { socioeconómicos }\end{array}$ & $\begin{array}{l}\text { Factores de } \\
\text { personalidad }\end{array}$ & $\begin{array}{c}\text { Factores } \\
\text { socioculturales }\end{array}$ \\
\hline$\overline{A 1(1)}$ & & .676 & & \\
\hline $\mathrm{A} 2(2)$ & & .761 & & \\
\hline $\mathrm{A} 3(3)$ & & .801 & & \\
\hline $\mathrm{A} 4(4)$ & & .575 & & \\
\hline $\mathrm{A} 5(5)$ & & .734 & & \\
\hline$A 6(6)$ & & .716 & & \\
\hline $\mathrm{A} 7(7)$ & & .773 & & \\
\hline A11(8) & & & .553 & \\
\hline $\mathrm{A} 12(9)$ & & & .805 & \\
\hline$A 13(10)$ & & & .795 & \\
\hline $\mathrm{A} 14(11)$ & & & .766 & \\
\hline$A 15(12)$ & & & .677 & \\
\hline $\mathrm{A} 16(13)$ & & & .735 & \\
\hline A17(14) & & & .622 & \\
\hline $\mathrm{A} 18(15)$ & & & & .527 \\
\hline $\mathrm{A} 19(16)$ & & & & .513 \\
\hline A20(17) & & .571 & & \\
\hline A21(18) & & .588 & & \\
\hline A22(19) & & & & .516 \\
\hline A23(20) & & & & .494 \\
\hline $\mathrm{A} 27(21)$ & .621 & & & \\
\hline A28(22) & .570 & & & \\
\hline A29(23) & .498 & & & \\
\hline A30(24) & .796 & & & \\
\hline A31(25) & .834 & & & \\
\hline A32(26) & .745 & & & \\
\hline A33(27) & .722 & & & \\
\hline A34(28) & .615 & & & \\
\hline A35(29) & .722 & & & \\
\hline A36(30) & .430 & & & \\
\hline A37(31) & .409 & & & \\
\hline $\begin{array}{c}\% \text { Varianza total } \\
\text { explicada }\end{array}$ & 18.36 & 12.75 & 8.36 & 4.94 \\
\hline$\alpha$ & .86 & .87 & .85 & .61 \\
\hline
\end{tabular}

Nota explicativa: los números correlativos en paréntesis corresponden a la versión final de la prueba adjuntada en el apéndice. 
Validez de criterio divergente

Para la validez de criterio divergente, se realizó una correlación de Pearson (r), correlacionando las dimensiones de deserción universitaria con las dimensiones de la escala adaptación a la universidad, obteniendo los siguientes datos: se encontraron correlaciones positivas bajas entre los factores académicos con adaptación social $(r=.223 ; p$ $<.001)$, con adaptación institucional $(r=338 ; p<.001)$ y adaptación académica $(r=368 ; p<.001)$, encontrándose con ello indicadores aceptables de validez convergente.

\section{Análisis de confiabilidad}

Para el análisis de ítems y de consistencia interna de los cuatro factores obtenidos mediante el análisis de componentes principales, que incluye la estructura factorial o dimensional. Está conformado por el factor socioeconómico, con los ítems 1, 2, 3, 4, 5, 6, 7, 20 y 21; la dimensión factor de personalidad, ítems 11, 12, 13, 14, 15, 16 y 17; la dimensión factor sociocultural, ítems 18, 19, 22 y 23; y la dimensión factor académico, con los ítems 27, 28, 29, 30, 31, 32, 33, $34,35,36$ y 37 . La dimensión factores socioeconómicos tuvo un valor de consistencia interna Alfa de Cronbach de .87; la media de respuesta a los ítems osciló entre 2.55 (ítem 6) y 3.00 (ítem 2). En la mayoría de los ítems, la media se encuentra por debajo de la media de la escala, que es de 3.0. Las desviaciones estándar en la mayoría de los ítems superan la unidad. Tal como se observa en la tabla 2, junto con estos estadísticos se calculó la correlación ítem-total y el Alfa de Cronbach si se elimina el ítem.

Tabla 2. Media ( $M)$, desviación típica (DT), correlación ítems total (Ci-T) y el Alfa de Cronbach si el ítem es eliminado $(\alpha)$ de la dimensión factores socioeconómicos

\begin{tabular}{ccccc}
\hline Reactivos & $\mathbf{M}$ & $\mathrm{DE}$ & $\mathrm{Ci}-\mathrm{T}$ & $\boldsymbol{\alpha}$ \\
\hline $\mathrm{A} 1(1)$ & 2.70 & 0.90 & .577 & .859 \\
$\mathrm{~A} 2(2)$ & 3.00 & 0.98 & .659 & .851 \\
$\mathrm{~A} 3(3)$ & 2.82 & 0.91 & .703 & .849 \\
$\mathrm{~A} 4(4)$ & 2.89 & 1.37 & .482 & .873 \\
$\mathrm{~A} 5(5)$ & 2.76 & 1.06 & .672 & .850 \\
$\mathrm{~A} 6(6)$ & 2.55 & 1.00 & .674 & .850 \\
$\mathrm{~A} 7(7)$ & 2.90 & 1.01 & .691 & .848 \\
$\mathrm{~A} 20(17)$ & 2.76 & 1.10 & .562 & .860 \\
$\mathrm{~A} 21(18)$ & 2.83 & 1.22 & .536 & .864 \\
\hline
\end{tabular}

En la dimensión factores de personalidad, se tuvo un valor de consistencia interna Alfa de Cronbach de .85; la media de respuesta a los ítems osciló entre 1.59 (ítem 11) y 1.97 (ítem 16). En todos los ítems, la media se encuentra abajo de la media de la escala, que es de 3.0. Las desviaciones estándar en todos los ítems son cercanas al valor de la unidad. Tal como se observa en la tabla 3, junto con estos estadísticos se calculó la correlación ítem-total y el Alfa de Cronbach si se elimina el ítem. 
Tabla 3. Media (M), desviación típica (DT), correlación ítems total (Ci-T) y el Alfa de Cronbach si el ítem es eliminado $(\alpha)$ de la dimensión factores de personalidad

\begin{tabular}{ccccc}
\hline Reactivos & $\mathbf{M}$ & $\mathbf{D E}$ & $\mathbf{C i - T}$ & $\boldsymbol{\alpha}$ \\
\hline A11(8) & 1.59 & 0.78 & .456 & .854 \\
A12(9) & 1.80 & 0.94 & .707 & .819 \\
A13(10) & 1.84 & 0.91 & .694 & .821 \\
A14(11) & 1.96 & 0.97 & .691 & .822 \\
A15(12) & 1.64 & 0.84 & .590 & .837 \\
A16(13) & 1.97 & 0.85 & .632 & .831 \\
A17(14) & 1.92 & 0.86 & .535 & .844 \\
\hline
\end{tabular}

La dimensión factores socioculturales tuvo un valor de consistencia interna Alfa de Cronbach de .61; la media de respuesta a los ítems osciló entre 1.47 (ítem 23) y 2.41 (ítem 22). En todos los ítems, la media se encuentra abajo de la media de la escala, que es de 3.0. Las desviaciones estándar en la mayoría de los ítems son cercanas al valor de la unidad. Tal como se observa en la tabla 4, junto con estos estadísticos se calculó la correlación ítem-total y el alfa de Cronbach si se elimina el ítem.

Tabla 4. Media (M), desviación típica (DT), correlación ítems total (Ci-T) y el alfa de Cronbach si el ítem es eliminado $(\alpha)$ de la dimensión factores socioculturales

\begin{tabular}{|c|c|c|c|c|}
\hline Reactivos & $\mathbf{M}$ & $\mathbf{D E}$ & $\mathbf{C i - T}$ & $\boldsymbol{\alpha}$ \\
\hline $\mathrm{A} 18(15)$ & 1.57 & 0.78 & .454 & .496 \\
\hline $\mathrm{A} 19(16)$ & 1.48 & 0.80 & .415 & .521 \\
\hline $\mathrm{A} 22(19)$ & 2.41 & 1.14 & .371 & .575 \\
\hline $\mathrm{A} 23(20)$ & 1.47 & 0.83 & .353 & .561 \\
\hline
\end{tabular}

La dimensión factores académicos tuvo un valor de consistencia interna Alfa de Cronbach de .86; la media de respuesta a los ítems osciló entre 3.22 (ítem 28) y 4.29 (ítem 37). En todos los ítems, la media se encuentra arriba de la media de la escala, que es de 3.0. Las desviaciones estándar en la mayoría de los ítems están arriba del valor de la unidad. Tal como se observa en la tabla 5, junto con estos estadísticos se calculó la correlación ítem-total y el Alfa de Cronbach si se elimina el ítem. 
Tabla 5. Media (M), desviación típica (DT), correlación ítems total (Ci-T) y el Alfa de Cronbach si el ítem es eliminado $(\alpha)$ de la dimensión factores académicos

\begin{tabular}{ccccc}
\hline Reactivos & $\mathbf{M}$ & DE & Ci-T & $\boldsymbol{\alpha}$ \\
\hline $\mathrm{A} 27(21)$ & 3.69 & 1.08 & .546 & .852 \\
$\mathrm{~A} 28(22)$ & 3.22 & 1.26 & .484 & .859 \\
$\mathrm{~A} 29(23)$ & 2.78 & 1.29 & .389 & .867 \\
$\mathrm{~A} 30(24)$ & 3.44 & 1.09 & .712 & .840 \\
$\mathrm{~A} 31(25)$ & 3.39 & 1.09 & .755 & .836 \\
$\mathrm{~A} 32(26)$ & 3.43 & 1.20 & .644 & .845 \\
$\mathrm{~A} 33(27)$ & 3.83 & 1.01 & .643 & .846 \\
$\mathrm{~A} 34(28)$ & 3.90 & 0.91 & .566 & .852 \\
$\mathrm{~A} 35(29)$ & 3.82 & 0.98 & .668 & .844 \\
$\mathrm{~A} 36(30)$ & 3.76 & 0.77 & .406 & .861 \\
$\mathrm{~A} 37(31)$ & 4.29 & 0.85 & .397 & .862 \\
\hline
\end{tabular}

Análisis inferencial. Para abundar en el presente estudio, se aplicaron pruebas no paramétricas para contrastar las dimensiones de deserción en función de las variables socioculturales, obteniendo los siguientes resultados: en la aplicación de la prueba U de Mann-Whitney, en función de género, no se encontraron diferencias estadísticas significativas; sin embargo, al aplicar dicha técnica en función de residencia se encontraron diferencias estadísticas en las dimensiones factores socioeconómicos $(z=-3.051 ; p=$ $.002 ; r=.16)$, factores de personalidad $(z=-3.352$; $p=.001 ; r=.18)$, y factores socioculturales $(z=-2.049$; $p=.040 ; r=.11$ ), siendo los estudiantes que residen en la zona rural quienes presentan mayores problemas en dichas dimensiones; los rangos promedios indican un efecto pequeño. Al aplicar la misma técnica, en función del tipo de institución donde estudio bachillerato, se encontraron diferencias estadísticas en factores socioeconómicos $(z=$ $-4.494 ; p<.001 ; r=.238$ ), siendo los estudiantes que salieron de bachillerato de una institución pública los que presentan mayores problemas socioeconómicos; los rangos promedios indican un efecto pequeño. En la aplicación de la prueba $\mathrm{H}$ de Kruskal-Wallis, en función de quien paga sus estudios universitarios, se encontraron diferencias estadísticas en la dimensión factores socioeconómicos $\left[X^{2}(2, N=353)=6.732\right.$; $\left.p=.032 ; E R_{2}=.019\right]$, siendo los estudiantes que pagan sus estudios universitarios con ingresos propios quienes presentan mayores problemas socioeconómicos.

\section{Discusión}

El problema de la deserción estudiantil universitaria es un tema de interés, tanto para los administradores educativos, los docentes, las universidades como para los Estados; y es de mayor impacto para los estudiantes debido a las consecuencias en su vida laboral y profesional. Existes diferentes definiciones de deserción educativa. Entre las más frecuentes, se tienen: "Deserción es el abandono de los estudios de forma temporal o permanente, producido por diversos factores"; "deserción es la falta de matrícula al inicio del semestre con la promoción que inició su carrera o el abandono de sus estudios y la inscripción con otra promoción, en otra carrera o en otra universidad" (Páramo \& Correa, 1999). Al estudiar la deserción de estudiantes universitarios, es de suma importancia tener claridad de que se trata de un problema educativo complejo, multifactorial (Álvarez, 1997) y prevalente en diferentes países. Se estima que entre 35 y $55 \%$ de estudiantes universitarios abandonan su carrera. También, que entre el 10 y $20 \%$ de los que ingresan a la universidad finalizan su carrera. Existen países como El Salvador, donde menos del $1 \%$ de los jóvenes que finalizan el bachillerato inician una carrera del nivel superior, aunque este problema también se observa en otros países, especialmente en los subdesarrollados y emergentes, indicando que el acceso a la universidad está condicionado por factores económicos, de personalidad, 
sociales, culturales; por el empleo de los padres, el trabajo de los estudiantes, la geografía de los países y la distancia de los centros universitarios, entre otros.

Tras el cumplimiento de las etapas estandarizadas en la construcción de una prueba, desde la perspectiva de la teoría clásica del test (Carretero-Dios, 2007), se tiene una escala que posee una validez de contenido con un coeficiente de concordancia de Kendal de .80, que indica alta concordancia entre los expertos sobre los reactivos y sus factores para medir deserción estudiantil universitaria. Por otra parte, el análisis factorial mediante componentes principales brindó indicadores adecuados para la validez de constructo de la prueba. Casi todos los ítems poseen una carga factorial muy superior al .40. La varianza total explicada supera el $44 \%$. En relación con la validez de criterio divergente, sus coeficientes de correlación entre las dimensiones de la escala de deserción universitaria y la de adaptación a la universidad son bajos, tal como se esperaba, indicando adecuada validez de criterio divergente. Referente a la confiabilidad mediante la consistencia interna Alfa de Cronbach, los análisis demostraron que los factores económicos, de personalidad y académicos presentan coeficientes superiores a .80; y el factor sociocultural brindó un coeficiente superior a .60. Estos resultados indican que la prueba posee adecuados coeficientes de validez de contenido, de constructo y de criterio, y buena confiabilidad, para medir la deserción estudiantil universitaria en El Salvador.

\section{Referencias}

Abarca-Rodríguez, A., \& Sánchez-Vindas, A. (2005). "La deserción estudiantil en la educación superior: el caso de la Universidad de Costa Rica". Revista Electrónica Actualidades Investigativas en Educación. Recuperado https://www.redalyc. org/articulo.oa?id=44759911

Álvarez, J.M. (1997). Etiología de un sueño. Bogotá: Universidad Autónoma de Colombia.

Ariza-Gasca, S.M., \& Marín-Arias, D.A. (2009). "Factores intervinientes en la deserción escolar de la Facultad de Psicología, Fundación Universitaria Los Libertadores". Tesis Psicológica. Recuperado de https://www.redalyc. org/articulo.oa?id=139013586006

Bolaños, F. (1985). "Estudios de opinión a un grupo de 61 desertores de la UNED". San José: UNED.

Cabrera, L., Bethencourt, J.T., Álvarez-Pérez, P., \& GonzálezAfonso, M. (2006). "El problema del abandono de
Ios estudios universitarios". Revista Electrónica de Investigación y Evaluación Educativa. Recuperado de https://ojs.uv.es/index. php/RELIEVE/article/view/4226 Carretero-Dios, H. \& Pérez, C. (2007). "Standards for the development and review of instrumental studies: considerations about test selection in psychological research". International Journal of Clinical and Health. Recuperado de http://www.aepc. es/ijchp/carretero_ es.pdf

González-Fiegehen, L.E. (2006). "Repitencia y deserción universitaria en América Latina". Recuperado de https:// www.researchgate.net/publication/275275302 Repitencia_y_desercion_en_America_Latina_2006-19

Durán, J. \& Díaz, G. (1990). “Análisis de la deserción estudiantil en la Universidad Autónoma Metropolitana". Revista de Educación Superior. Recuperado de http:// publicaciones. anuies.mx/pdfs/revista/Revista74_ S1A3ES.pdf

González-Fiegehen, L.E. (2005). "Estudio sobre a repitencia y deserción en la educación superior chilena". Recuperado de https://www.inacap.cl/ tportal/portales/tp4964b0e 1bk102/uploadımg/File/ REPITENCIA_DESERCION_L_E_Gonzalez_2005.pdf

González-Fiegehen, L.E., \& Espinoza-Días, O. (2008). "Deserción en educación superior en América Latina y el Caribe". Paideia. Recuperado de http:// www.revistapaideia. cl/index.php/PAIDEIA/article/ view/71/65

Himmel, E. (2005). "Modelos de análisis de la deserción estudiantil en la educación superior". Revista Calidad en la Educación. Recuperado de https:// calidadenlaeducacion .cl/index.php/rce/article/view/409

Latiesa, M. (1992). La deserción universitaria. Madrid: Centro de Investigaciones sociológicas.

Mateus-Rodríguez, M., Herrera-Hernández, C., Perilla-Suárez, C., Parra-Quecan, G., \& Vera-Maldonado, A. (2011). "Factores presentes en la deserción universitaria en la Facultad de Psicología de la Universidad de San Buenaventura, Sede Bogotá en el periodo comprendido entre 1998-2009. Psychologia: Avances de la Disciplina. Recuperado de https://www.redalyc. org/articulo.oa?id=297224114011

Montero, I. \& León, O.G. (2007). “A guide for naming research studies in Psychology". International Journal of Clinical and Health Psychology. Recuperado de https://www. redalyc.org/html/337/33770318/

Montero-Rojas, E., Villalobos-Palma, J., \& Valverde-Bermúdez, A. (2007). "Factores institucionales, pedagógicos, 
psicosociales y sociodemográficos asociados al rendimiento académico en la Universidad de Costa Rica: un análisis multinivel". RELIEVE. Recuperado de https://www.redalyc.org/articulo.oa?id=91613205

Mori-Sánchez, M. P. (2012). Deserción universitaria en estudiantes de una universidad privada de Iquitos. Revista Digital de Investigación en Docencia Universitaria. Recuperado de http://hdl.handle. net/10757/325224

Páramo, G.J., \& Correa, C.A. (1999). "Deserción estudiantil universitaria. Conceptualización". Revista Académica Universidad Eafit. Recuperado de http://publicaciones. eafit.edu.co/index.php/revista-universidad-eafit/ article/view/1075
Pérez, M., Bravo, O., \& Isabeles, S. (2008). "Principales causas de deserción escolar de la Facultad de Lenguas Extranjeras de la Universidad de Colima de la generación 2004, 2005 y 2006". Memorias del IV Foro Nacional de Estudios en Lenguas (FONAEL 2008). Quintana Roo, México: Universidad de Quintana Roo.

Rodríguez Lagunas, J., \& Hernández Vázquez, J.M. (2008). "La deserción universitaria en México. La experiencia de la Universidad Autónoma Metropolitana Campus Iztapalapa". Instituto de investigación en educación. Actualidades investigativas en educación. Recuperado de https://revistas.ucr.ac.cr/index.php/aie/article/ view /9308/17772

Apéndice

\section{Escala de deserción estudiantil universitaria}

\begin{tabular}{|c|c|c|c|c|c|c|}
\hline n. ${ }^{\circ}$ & Reactivos & 1 & 2 & 3 & 4 & 5 \\
\hline 1 & He tenido dificultades con los pagos de la universidad. & Nunca & Rara vez & Algunas veces & Casi siempre & Siempre \\
\hline 2 & $\begin{array}{l}\text { He tenido dificultades económicas con los gastos de libros, } \\
\text { folletos, entre otros. }\end{array}$ & Nunca & Rara vez & Algunas veces & Casi siempre & Siempre \\
\hline 3 & $\begin{array}{l}\text { He tenido dificultades económicas con los gastos de } \\
\text { materiales para hacer mis actividades académicas. }\end{array}$ & Nunca & Rara vez & Algunas veces & Casi siempre & Siempre \\
\hline 4 & $\begin{array}{l}\text { Mis padres han tenido dificultades económicas para ayudarme } \\
\text { con los gastos universitarios (cuotas, materiales, libros). }\end{array}$ & Nunca & Rara vez & Algunas veces & Casi siempre & Siempre \\
\hline 5 & $\begin{array}{l}\text { He tenido dificultades para costear mi alimentación (desayuno, } \\
\text { almuerzo, cena) en la universidad o sus alrededores. }\end{array}$ & Nunca & Rara vez & Algunas veces & Casi siempre & Siempre \\
\hline 6 & $\begin{array}{l}\text { He tenido dificultades para costear mis gastos de transporte } \\
\text { para asistir a la universidad. }\end{array}$ & Nunca & Rara vez & Algunas veces & Casi siempre & Siempre \\
\hline 7 & $\begin{array}{l}\text { El dinero ha sido un impedimento para el desarrollo de mis } \\
\text { actividades académicas dentro y fuera de la universidad. }\end{array}$ & Nunca & Rara vez & Algunas veces & Casi siempre & Siempre \\
\hline 8 & He tenido dificultades para adaptarme a la universidad. & Nunca & Rara vez & Algunas veces & Casi siempre & Siempre \\
\hline 9 & $\begin{array}{l}\text { Las dificultades emocionales han interferido en mi proceso } \\
\text { educativo universitario. }\end{array}$ & Nunca & Rara vez & Algunas veces & Casi siempre & Siempre \\
\hline 10 & $\begin{array}{l}\text { Los problemas interpersonales han interferido en mi proceso } \\
\text { educativo universitario. }\end{array}$ & Nunca & Rara vez & Algunas veces & Casi siempre & Siempre \\
\hline 11 & $\begin{array}{l}\text { Las situaciones de frustración han interferido en mi proceso } \\
\text { educativo universitario. }\end{array}$ & Nunca & Rara vez & Algunas veces & Casi siempre & Siempre \\
\hline 12 & $\begin{array}{l}\text { Durante el proceso educativo universitario he tenido problemas } \\
\text { con mi autoestima. }\end{array}$ & Nunca & Rara vez & Algunas veces & Casi siempre & Siempre \\
\hline 13 & $\begin{array}{l}\text { Durante el proceso educativo universitario, he tenido } \\
\text { dificultades con el aprendizaje. }\end{array}$ & Nunca & Rara vez & Algunas veces & Casi siempre & Siempre \\
\hline
\end{tabular}




\begin{tabular}{|c|c|c|c|c|c|c|}
\hline 14 & $\begin{array}{l}\text { Durante el proceso educativo universitario, presento baja } \\
\text { motivación hacia mis estudios. }\end{array}$ & Nunca & Rara vez & Algunas veces & Casi siempre & Siempre \\
\hline 15 & $\begin{array}{l}\text { Durante el proceso educativo universitario, he tenido conflictos } \\
\text { con mis compañeros. }\end{array}$ & Nunca & Rara vez & Algunas veces & Casi siempre & Siempre \\
\hline 16 & $\begin{array}{l}\text { Durante el proceso educativo universitario, he tenido conflictos } \\
\text { con mis profesores. }\end{array}$ & Nunca & Rara vez & Algunas veces & Casi siempre & Siempre \\
\hline 17 & $\begin{array}{l}\text { La situación económica del país me ha afectado para no } \\
\text { continuar con mis estudios universitarios. }\end{array}$ & Nunca & Rara vez & Algunas veces & Casi siempre & Siempre \\
\hline 18 & $\begin{array}{l}\text { La situación laboral del país me ha afectado para continuar } \\
\text { con mis estudios universitarios. }\end{array}$ & Nunca & Rara vez & Algunas veces & Casi siempre & Siempre \\
\hline 19 & $\begin{array}{l}\text { La situación de violencia e inseguridad del país me ha } \\
\text { afectado para continuar con mis estudios universitarios. }\end{array}$ & Nunca & Rara vez & Algunas veces & Casi siempre & Siempre \\
\hline 20 & Tengo falta de compromiso con mi formación académica. & Nunca & Rara vez & Algunas veces & Casi siempre & Siempre \\
\hline 21 & El inicio de clases en la universidad fue... & Deficiente & Regular & Bueno & Muy bueno & Excelente \\
\hline 22 & El horario de clases que tuve fue... & Deficiente & Regular & Bueno & Muy bueno & Excelente \\
\hline 23 & $\begin{array}{l}\text { Mi participación en actividades extra-curriculares (ej.: } \\
\text { voluntariado en proyectos, instructoría, deportes, proyección } \\
\text { social, etc.) fue... }\end{array}$ & Deficiente & Regular & Bueno & Muy bueno & Excelente \\
\hline 24 & $\begin{array}{l}\text { La relación con el personal administrativo de la universidad } \\
\text { fue... }\end{array}$ & Deficiente & Regular & Bueno & Muy bueno & Excelente \\
\hline 25 & $\begin{array}{l}\text { La orientación de los servicios de la universidad y la cultura } \\
\text { universitaria fue... }\end{array}$ & Deficiente & Regular & Bueno & Muy bueno & Excelente \\
\hline 26 & $\begin{array}{l}\text { La información que el personal universitario me brindó acerca } \\
\text { de mi carrera fue... }\end{array}$ & Deficiente & Regular & Bueno & Muy bueno & Excelente \\
\hline 27 & La forma en la que el profesor imparte clases fue... & Deficiente & Regular & Bueno & Muy bueno & Excelente \\
\hline 28 & La interacción con mis compañeros es/fue... & Deficiente & Regular & Bueno & Muy bueno & Excelente \\
\hline 29 & La relación con mis profesores de clase es... & Deficiente & Regular & Bueno & Muy bueno & Excelente \\
\hline 30 & Mi rendimiento académico es/fue... & Deficiente & Regular & Bueno & Muy bueno & Excelente \\
\hline 31 & $\begin{array}{l}\text { Las expectativas que actualmente tengo de la carrera, con } \\
\text { respecto a mi formación como profesional, son... }\end{array}$ & Deficiente & Regular & Bueno & Muy bueno & Excelente \\
\hline
\end{tabular}

\section{Dimensiones}

\begin{tabular}{|l|l|}
\hline Factores socioeconómicos & Ítems $1,2,3,4,5,6,7,17,18$ \\
\hline Factores de personalidad & Ítems $8,9,10,11,12,13,14$ \\
\hline Factores socioculturales & Ítems $15,16,19,20$ \\
\hline Factores académicos & Ítems $21,22,23,24,25,26,27,28,29,30,31$ \\
\hline
\end{tabular}

